

\title{
Velocity Distribution and Base Pressure Analysis of Under Expanded Nozzle Flow at Mach 1.0
}

\author{
Ambareen Khan ${ }^{1}$, Nurul Musfirah Mazlan ${ }^{1,}{ }^{*}$, Mohd Azmi Ismail ${ }^{2}$ \\ School of Aerospace Engineering, Engineering Campus, Universiti Sains Malaysia, 14300, Nibong Tebal, Pulau Pinang, Malaysia \\ 2 School of Mechanical Engineering, Engineering Campus, Universiti Sains Malaysia, 14300, Nibong Tebal, Pulau Pinang, Malaysia
}

\section{ARTICLE INFO}

\section{Article history:}

Received 27 July 2021

Received in revised form 2 January 2022

Accepted 8 January 2022

Available online 7 February 2022

\section{Keywords:}

Annular rib; Velocity distribution; Base Pressure; Expanded Nozzle

\section{ABSTRACT}

This paper discusses velocity distribution and base pressure control using annular ribs. The annular ribs are located in the enlarged duct. Its impact on the velocity distribution and base pressure changes from a converging nozzle at the sonic Mach number is investigated numerically using ANSYS software. The rib's height is fixed to $3 \mathrm{~mm}$ while the rib's height is varied from $1 \mathrm{~mm}$ to $2 \mathrm{~mm}$. The simulation is performed by placing the rib at various locations in the duct namely 1D, 2D, 3D and 4D and at Nozzle Pressure Ration (NPR) varied from 1.5 to 5 . The changes in base pressure $\left(P_{b}\right)$ and velocity variation inside the duct are studied. The presence of rib in the duct causes disturbance in the flow field hence increases the $P_{b}$. The tendency of $P_{b}$ to increase is observed as the height of the rib increases. Varying the NPR from 1.5 to 3 reduced $P_{b}$ due to shock wave formation. The NPR increases with $P_{b}$ as the flow undergoes under-expansion due to the expansion fan at the nozzle exit. The changes in $\mathrm{Pb}_{\mathrm{b}}$ are consistent with velocity distribution inside the duct. The increase in turbulent kinetic energy is more significant due to the presence of rib, as compared to duct without rib. For lower NPR, the velocity is found to be highest at the axial direction of the duct while the highest velocity is found just ahead of the nozzle exit at higher NPR.

\section{Introduction}

The effect of compressible flow is dominant in any applications related to high-speed flow such as in a high-speed aircraft, rockets, reentry vehicles, projectiles, and external ballistics. During the subsonic and supersonic flow regimes, the flow is compressible, and the air density varies. The variations in the density need to be considered while designing any high-speed vehicle. At a very high Mach number, aerodynamic heating becomes a severe problem that needs to be addressed. The aerodynamics at high Mach numbers is different from the supersonic flow. The linearised theory cannot be used at transonic Mach numbers.

During the linearised theory evolution, the second-order terms were neglected as their order of magnitude was very small compared to the first-order terms. However, this assumption fails when the Mach number is much closer to unity. In this research paper, the transonic flow was studied

\footnotetext{
* Corresponding author.

E-mail address: nmusfirah@usm.my

https://doi.org/10.37934/arfmts.92.1.177189
} 
through the internal flow method, where the flowing gas from a converging nozzle is suddenly expanded into a circular duct. Due to sudden expansion, the flowing gas is separated while exiting from the nozzle and forming a wake region at the base. The jet pump action in these flows leads to base corner flows where the base pressure is low, as illustrated in Figure 1.



Fig. 1. Detailed view of a Suddenly Expanded flow

The lowest pressure at corners causes base drag in the wake region. This low pressure may account for up to $60 \%$ of the total drag force. It has to be considered at the design stage and needs urgent attention to augment its magnitude and, hence, optimise the aerospace vehicles' aerodynamic shape. The pressure control at the duct's corner when the nozzle's flow exits can be achieved using several methods. Active and passive control methods are often adopted to increase base pressure [1-4]. Although active control is found to be the most efficient method to control the base pressure, the contribution of the passive method to control the base pressure cannot be deceived.

The experimental studies using internal flow test setup have many advantages over exterior ballistics tests conducted in the ballistics range for Shells, unguided rockets, bombs, and missiles. Mechanisms like stings used to support the model are eliminated and important for external flow while performing the internal flow experimental analysis. The use of an internal flow setup has the advantage of static pressure. The whole temperature of surface body measurement can be performed in the wake region corner and at the expansion portion's inlet section. The tests conducted in such a way are significant as they provide theoretical predictions accurately [5-15]. However, the experimental setup is costly. Thus, numerical simulation becomes an advantage to simulate the flow field of the high-speed flow in detail.

Simulation studies on the passive method in controlling the base pressure are still lacking. Khan et al., [16] performed experimental and numerical simulations for Mach 1 to 2.5. They found that the experimental and numerical simulation results of base pressure match and findings are within $10 \%$ error which is agreeable. Khan et al., [17] also performed a research study on the passive method using a rectangular rib shape with an area ratio of 4 . They found that the location of the rib is a key role in regulating base pressure. In experimental research done by Khan et al., [18], pressure loss and wall pressure were studied for Mach 1 and Mach 1.2 for area ratio 7.84 for a fixed L/D ratio of 8 . From their research, pressure loss is around $30 \%$ at low Mach numbers, and wall pressure is most lacking at NPR 7 and highest at NPR 1.5. Khan et al., [19] did a numerical simulation of a suddenly expanded duct of diameter $22 \mathrm{~mm}$. The rectangular rib was placed at 1D to 4D. The study's findings indicate that 3D or 4D is the most optimum location of the rib at which the base pressure increases. Khan et al., [20] have written a review on passive control in which they have found that passive management is effective whenever a favourable pressure gradient is maintained. 
Although the studies mentioned above have found that the passive rib is effective in managing the pressure gradient, however, the experimental and simulation works performed did not discuss in detail the flow behaviour particularly the velocity and pressure changes inside the duct. Therefore, to observe the changes, this work is performed to provide a comprehensive analysis specifically on the velocity and pressure distribution of the flow field inside the duct due to the effect of the rib locations and rib height to control the base pressure. To achieve the objective, the locations and the height of the rib are varied. The variation of Nozzle Pressure Ratio (NPR) also will be investigated as the increases of NPR is hypothesised to influence flow expansion thus create different flow phenomena. The present research is undertaken to understand the nature of flow behaviours in the suddenly expanded duct; and the physics behind these phenomena.

\section{Methodology}

Figure 2 shows a physical model of the nozzle and duct with the rib. The nozzle and duct are modelled as circular axis-symmetrically. The ratio between width $(\mathrm{W})$ and height $(\mathrm{H})$ of the rib are $3: 1$ and $3: 2(\mathrm{~W}: \mathrm{H})$. The nozzle has an inlet diameter of $30 \mathrm{~mm}$, and the outlet diameter is $10 \mathrm{~mm}$. The duct's length is $96 \mathrm{~mm}$, and the duct's outlet diameter is $16 \mathrm{~mm}$. The ratio of nozzle exit area to duct area of the model is 2.56 .

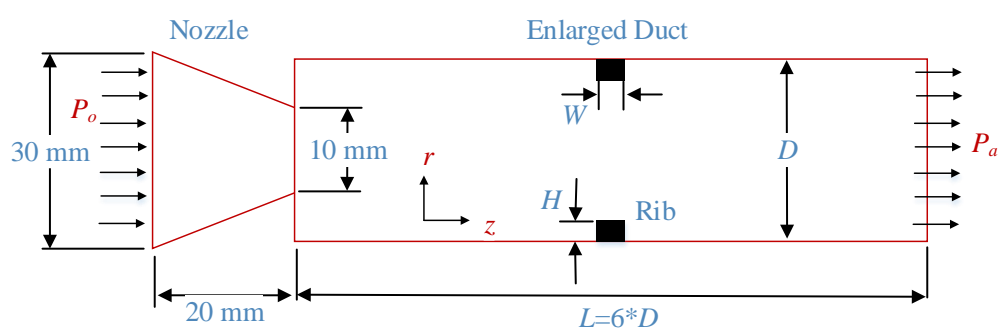

Fig. 2. Nozzle and duct arrangement with rib

The nozzle and duct were meshed using rectangular unstructured mesh. It is worth mentioning that the nozzle is a converging type that has a continuous decreasing area. Therefore, the unstructured mesh was the most suitable mesh to accommodate the effect at the wall and at the throat. Initially, the mesh independence analysis was performed for three types of mesh namely coarse, medium and fine. The course mesh has 42202 elements, the medium mesh has 47988 elements and the fine mesh has 53492 elements. For each type of mesh, the ratio between base pressure and atmospheric pressure over each nozzle pressure ratio (NPR) is compared as shown in Figure 3. Based on the comparison, it is found that medium-mesh provides closer results to fine mesh. Therefore, the medium mesh (47988 elements) was selected considering the tolerable computational time to complete one simulation compared to fine mesh. 


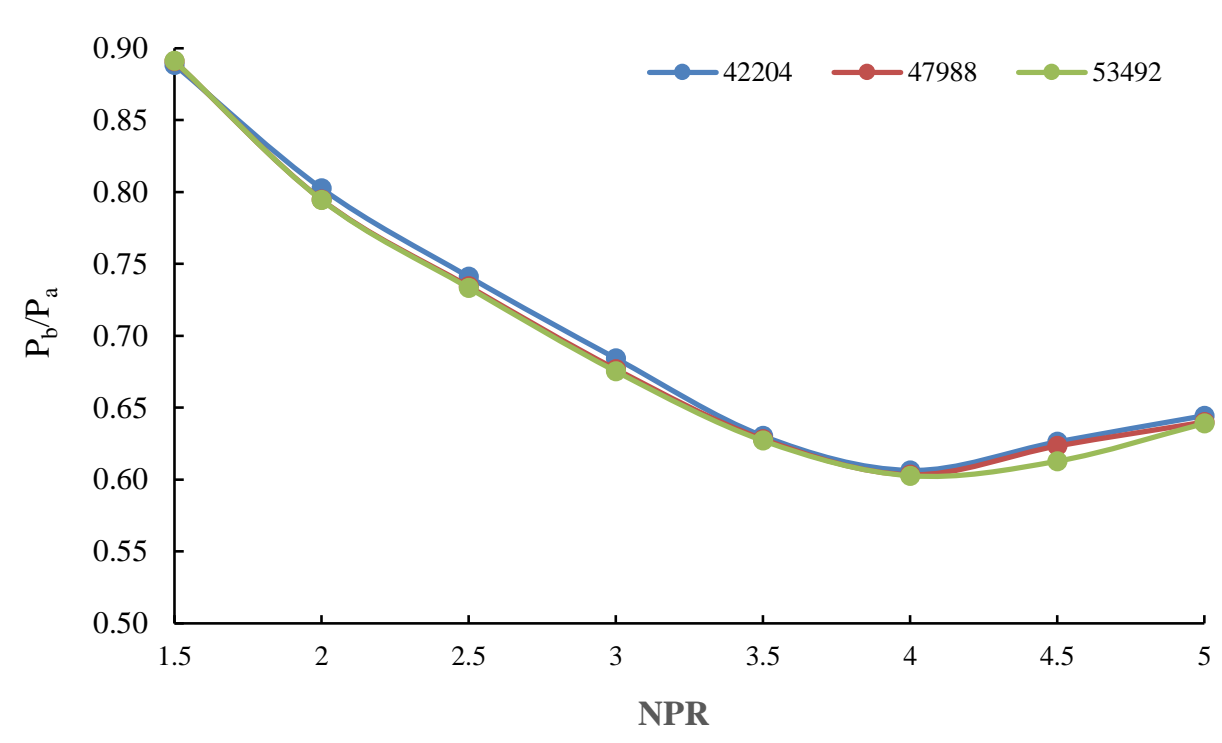

Fig. 3. Three different element size having converged values

The nozzle flow in this study is considered turbulent; hence, the $k-\varepsilon$ standard model is applied for the compressible flow field. The flowing equations represent the turbulent flow field most appropriately.

Continuity equation for the compressible flow (density $\rho$ based) with the steady-state condition in the 2-dimensional cylindrical coordinate system:

$$
\frac{1}{r} \frac{\partial(\rho r u)}{\partial r}+\frac{\partial(\rho v)}{\partial z}=0
$$

The time-averaged axial $z$-momentum equation representing $u$ velocity of flow is given by:

$$
\frac{1}{r} \frac{\partial(\rho r u u)}{\partial z}+\frac{1}{r} \frac{\partial(\rho v u)}{\partial r}=-\frac{\partial p}{\partial r}+\left(\mu+\mu_{t}\right) \frac{\partial}{\partial z}\left[2 \frac{\partial u}{\partial z}-\frac{2}{3}(\nabla \cdot \vec{v})\right]+\left(\mu+\mu_{t}\right) \frac{\partial}{\partial r}\left[\frac{\partial u}{\partial r}+\frac{\partial v}{\partial z}\right]
$$

The radial $r$-momentum equation for $v$ velocity

$$
\begin{aligned}
\frac{1}{r} \frac{\partial(\rho r u v)}{\partial z}+\frac{1}{r} & \frac{\partial(\rho v v)}{\partial r} \\
& =-\frac{\partial p}{\partial r}+\left(\mu+\mu_{t}\right) \frac{\partial}{\partial r}\left[\left(2 \frac{\partial u}{\partial z}-\frac{2}{3}(\nabla \cdot \vec{v})\right)\right]+\left(\mu+\mu_{t}\right) \frac{\partial}{\partial z}\left[\left(\frac{\partial u}{\partial r}+\frac{\partial v}{\partial z}\right)\right] \\
& -2 \frac{\left(\mu+\mu_{t}\right) v}{r^{2}}+\frac{2}{3} \frac{1}{r}\left(\mu+\mu_{t}\right)(\nabla \cdot \vec{v})
\end{aligned}
$$

The term $\vec{v}$ in Eq. (2) and (3) is given by:

$$
\nabla \cdot \vec{v}=\frac{\partial u}{\partial z}+\frac{\partial v}{\partial r}+\frac{v}{r}
$$

Here, $\mu$ is viscosity and $\mu_{o}$ is the reference viscosity value in $\mathrm{kg} / \mathrm{m}-\mathrm{s}, T$ is static temperature, and $T_{o}$ is the reference temperature in K. $S$ is Sutherland constant depending upon effective temperature. 
The quantity $\left(\frac{k}{C_{p}}+\frac{\mu_{t}}{P r_{t}}\right)$ represents the useful thermo-physical property of fluid where $\mathrm{k}$ is the thermal conductivity in $\mathrm{W} / \mathrm{m}^{2} \mathrm{k}, C_{p}$ is specific heat capacity in $\mathrm{KJ} / \mathrm{kg}-\mathrm{K}, \mu_{t}$ is turbulent viscosity in $\mathrm{kg} / \mathrm{m}-\mathrm{s}$, and $P r_{t}$ is the Prandt l number for turbulent flow

The $\mathrm{K}-\varepsilon$ turbulence model is one among the famously used model which provides economy, robustness, and sufficient accuracy for many kinds of flow situations. The k- $\varepsilon$ turbulence model employed in this work is available in ANSYS Fluent software. The turbulent kinetic energy, i.e., Kequation, is given by:

$$
\frac{\partial(\rho u \mathrm{~K})}{\partial z}+\frac{1}{r} \frac{\partial(\rho v \mathrm{~K})}{\partial r}=\frac{\partial}{\partial z}\left[\left(\mu+\frac{\mu_{t}}{\sigma_{k}}\right) \frac{\partial \mathrm{K}}{\partial z}\right]+\frac{1}{r} \frac{\partial}{\partial r}\left[r\left(\mu+\frac{\mu_{t}}{\sigma_{k}}\right) \frac{\partial \mathrm{K}}{\partial r}\right]-\rho \varepsilon+G
$$

The term $\sigma_{k}$ is the turbulent Prandtl number for $\mathrm{K}$, and $\varepsilon$ is turbulent kinetic energy dissipation rate, and $G$ is turbulence generation term given by:

$$
G=\mu_{t}\left(\frac{\partial u_{i}}{\partial x_{j}}+\frac{\partial u_{j}}{\partial x_{i}}\right) \frac{\partial u_{i}}{\partial x_{j}}-\frac{2}{3} k \delta_{i j} \frac{\partial u_{i}}{\partial x_{j}}
$$

The kinetic energy of turbulence dissipation, i.e., $\varepsilon$-equation is given by:

$$
\frac{\partial(\rho u \varepsilon)}{\partial z}+\frac{1}{r} \frac{\partial(\rho v \varepsilon)}{\partial r}=\frac{\partial}{\partial z}\left[\left(\mu+\frac{\mu_{t}}{\sigma_{\varepsilon}}\right) \frac{\partial \varepsilon}{\partial z}\right]+\frac{1}{r} \frac{\partial}{\partial r}\left[r\left(\mu+\frac{\mu_{t}}{\sigma_{\varepsilon}}\right) \frac{\partial \varepsilon}{\partial r}\right]-C_{1} f_{1}\left(\frac{\varepsilon}{\mathrm{K}}\right) G-C_{2} f_{2}\left(\frac{\varepsilon^{2}}{\mathrm{~K}}\right)
$$

The term $\mu_{t}=\rho f_{\mu} C_{\mu} k^{2} / \varepsilon$ represents turbulent viscosity, the value of $C_{\mu}, C_{1}, C_{2}, f_{\mu}, \sigma_{k}, \sigma_{\varepsilon}$ are arbitrary constants.

Understanding the physics of flow inside the duct requires the setup to be validated with the results of Rathakrishnan [21]. In his experimental work, five ribs were placed at the duct's central position, as shown in Figure 4. The variation of base pressure $\left(P_{b}\right)$ for various NPR and length-todiameter (L/D) was compared with results obtained in Rathakrishnan [21] for the setup with and without the rib (Figure 5). For the comparison purpose, the rib with the ratio of 3:3 (W: H) was used. The comparison shows that the simulation results are within a $10 \%$ agreeable error difference with Rathakrishnan [21].

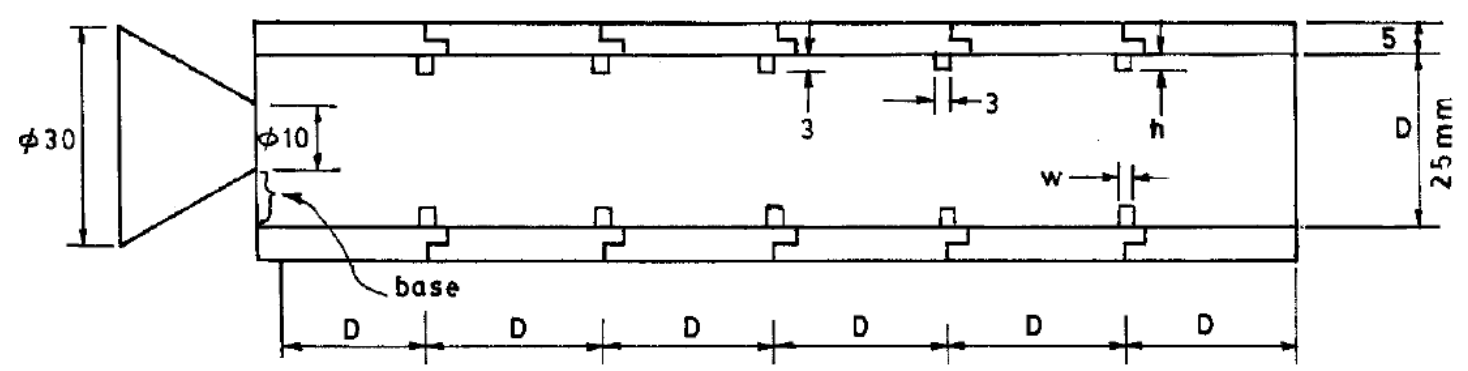

Fig. 4. Duct with five ribs used by Rathakrishnan [21] for experimental study 


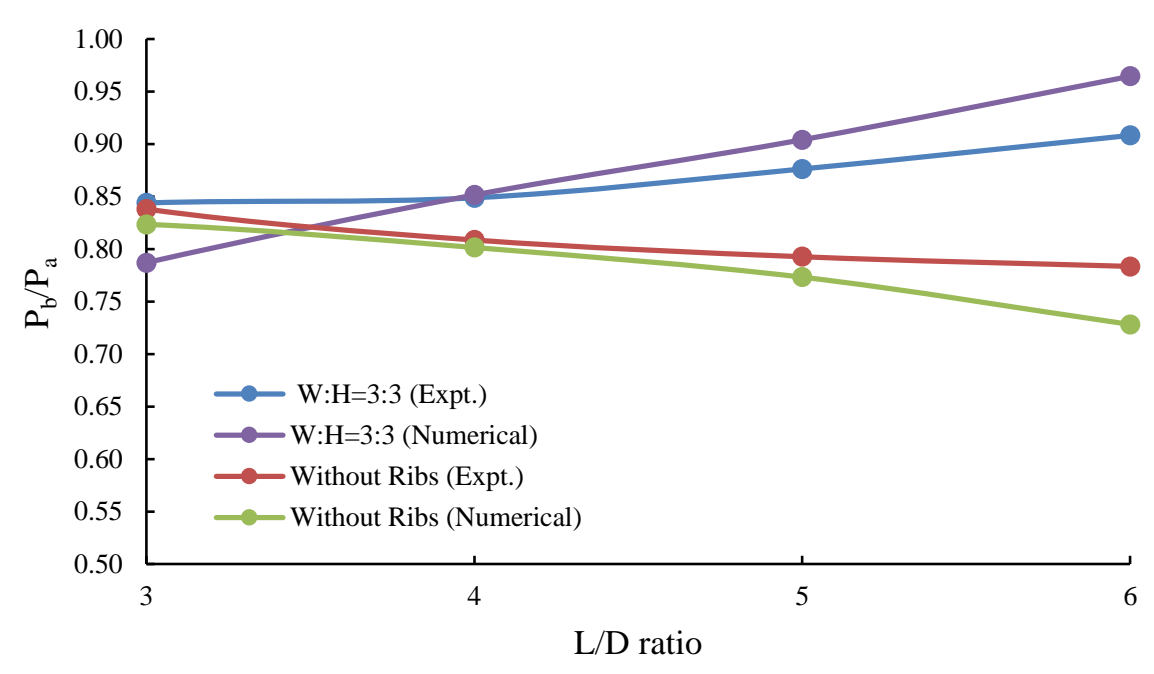

Fig. 5. Comparison of experimental and numerical results

\section{Results}

This section discusses flow behaviour in the suddenly expanded duct as it exits through a sonic nozzle. The circular duct diameter is $16 \mathrm{~mm}$, and the length-to-diameter ratio is 6 . The NPR is varied from 1.5 to 5 . The rectangular rib passive control is used. The width: height (W: $\mathrm{H}$ ) ratio of the rib were $3: 1$ and 3:2. These two ribs are numerically studied one at a time. The pressure distribution and velocity variation throughout the duct are studied at $1 \mathrm{D}$ and $2 \mathrm{D}$ locations in detail.

\subsection{Base Pressure Distribution}

Figure 6 shows the recirculation zones at the corner of the base and rib at NPR $=2.5$. Rib in both the streamlines is placed at 1D, i.e., at $16 \mathrm{~mm}$ away from the base. The aspect ratio in one case is 3:1, and in another, it is fixed at 3:2 at the exact location. It is evident that only one vortex, i.e., the primary vortex, can be seen when no rib is attached to the wall. When the rib is attached, primary and secondary vortices at the base corner and at the rib corner can be seen.

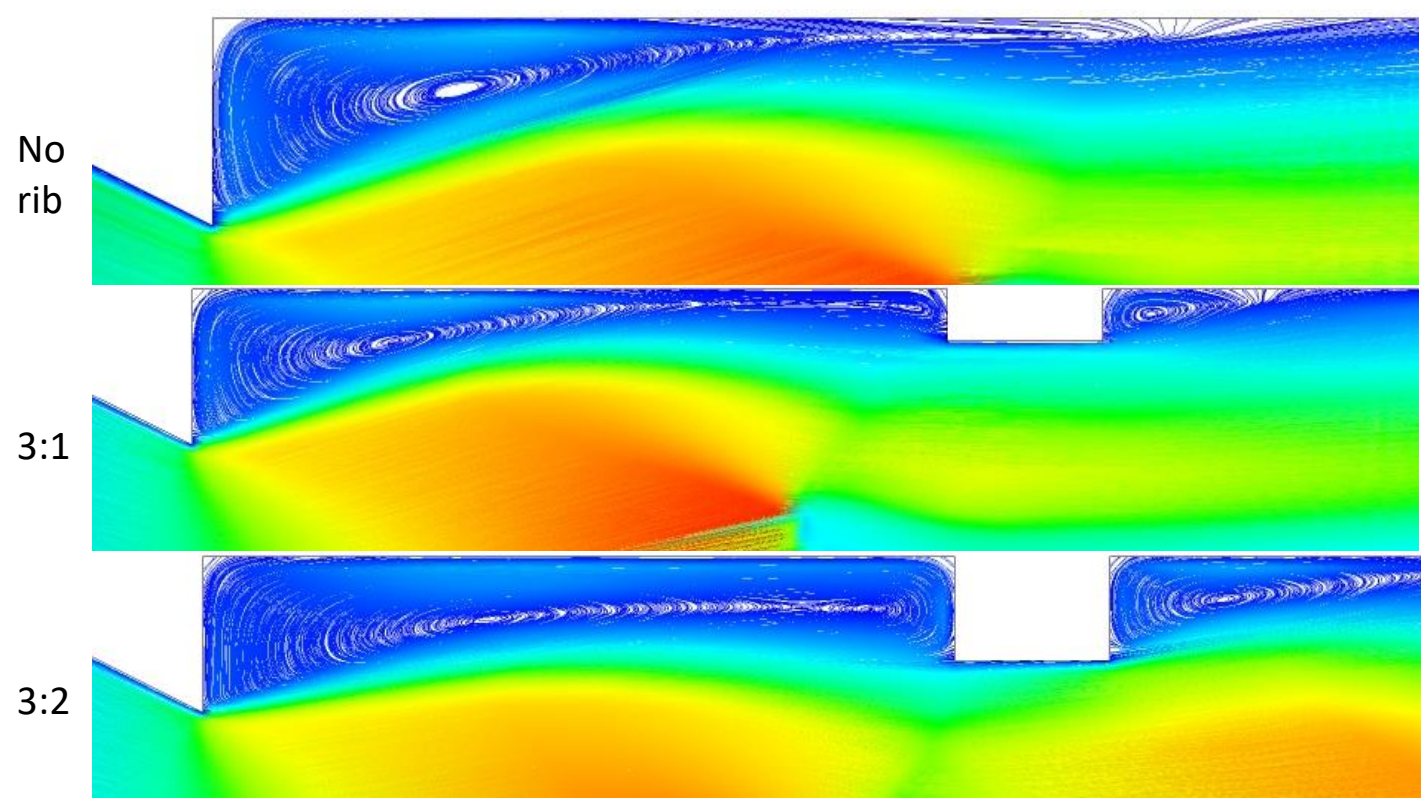

Fig. 6. Streamlines showing recirculation zones 
Additionally, for rib with a 3:1 aspect ratio, the primary vortex is smaller, and the secondary vortex is formed compared to the duct with no rib. With an increase in aspect ratio from 3:1 onwards, the primary vortex becomes smaller and the secondary vortex more enormous. However, there shall be a limit to the rib's height, which should not be increased as the flow passage cross-section area reduces. Contrary to this, with vortices at the rib corner, vibrations may get induced, which are not favourable.

Figure 7 shows the effect of the rib position on the base pressure $\left(P_{b}\right)$. The rib is located at $1 D$, 2D, 3D, and 4D. The rib height is $1 \mathrm{~mm}$ and $2 \mathrm{~mm}$. The figure clearly shows that the base pressure increases slowly by positioning the rib from 1D to 4D at a 3:1 aspect ratio. When the rib height is increased to $2 \mathrm{~mm}$, i.e., for a 3:2 aspect ratio, the base pressure is seen to jump at the exact location compared to the 3:1 location. With the presence of rib, the mass reentry into the primary vortex gets disturbed. The reattachment point plays a significant role in this mass reversal. If the rib is placed before the reattachment point, the backward fluid flow in the corner reduces, hence the base pressure will be lower than the duct without rib. The increment of base pressure also depends upon the rib's height. As the rib's height increases, the flow tends to get in touch with the rib corner due to the increased height causes the flow to reverse and leads to higher base pressure. The higher base pressure is favourable as it helps in reducing the base drag.



Fig. 7. Effect of rib location and height on base pressure

Figure 8 depicts a comparison of base pressure variation between case without rib and case with rib when placed at $1 \mathrm{D}$ and $2 \mathrm{D}$ as NPR increases from 1.5 to 5 . For all test cases, the base pressure decreases initially and increases as the NPR reaches 3 . The rib's aspect ratio is at 3:1. In the investigation, the impact of the shear layer, reattachment point, level of expansion, passive control using rib (location and geometry), and NPR have a promising role. The pressure difference, i.e., NPR, causes the flow to expand in the duct, wherein the reattachment length increases directly for an increase in the stagnation pressure. Initially, the base pressure reduces at NPR 1.5 to 2.5 or 3 . This is due to shock waves ahead of the nozzle, and the flow has not achieved the sonic condition when the NPR is 1.5. The pressure in the atmosphere is lower than that at the exit; hence, adverse pressure exists. As the adverse pressure reduces due to increases in NPR, the control effectiveness for 2D rib is found to increase the base pressure. For 1D rib location, the control effectiveness tries to reduce 
the base pressure. At this position, the rib acts as a barrier to the flow reversal into the corner recirculation zone.

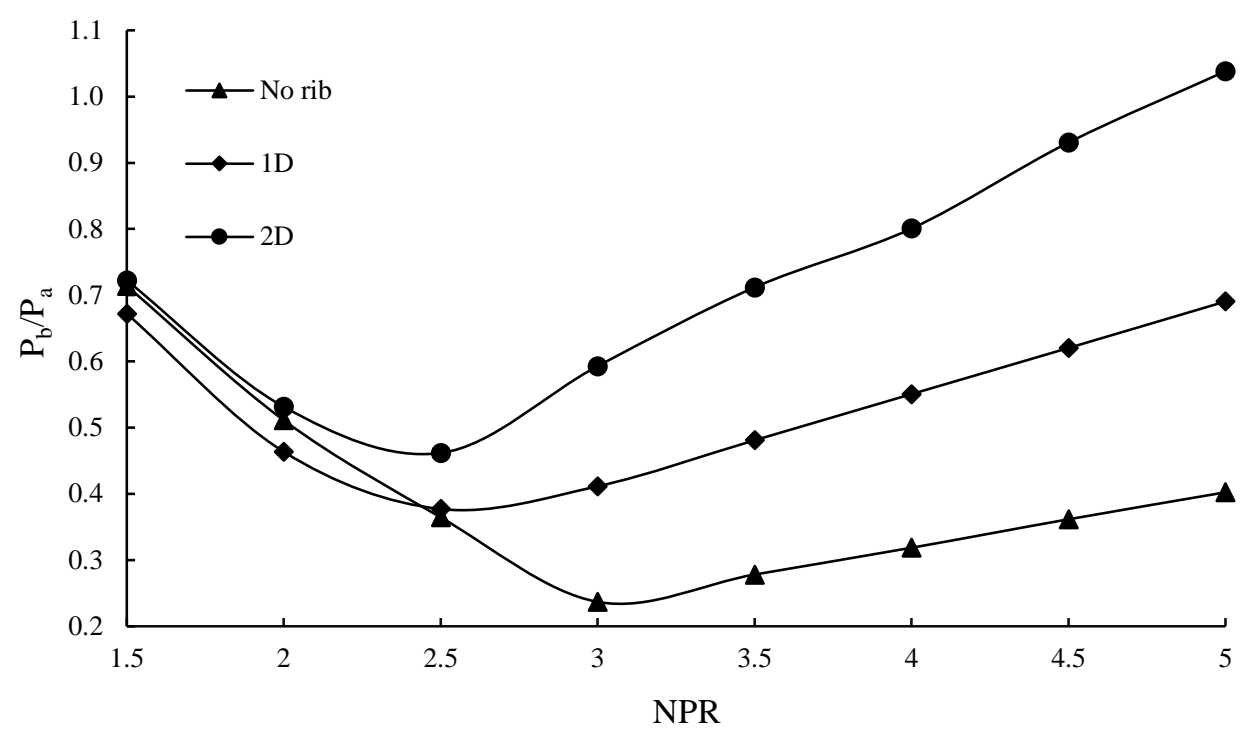

Fig. 8. Variation in base pressure with an increase in NPR from 1.5 to 5 for different cases at a fixed aspect ratio of rib 3:1

Furthermore, the base pressure is reduced than in duct without rib. However, with continuous increment in NPR, the flow undergoes under expansion where there will be an expansion fan at the nozzle exit. The adverse pressure gradient reduces significantly, and hence the passive control effectiveness is visible in both the cases of rib location at remaining NPR's. At the 2D location, the percentage increase in the base pressure becomes more dominant at higher NPR as the reattachment length comes closer to the $2 \mathrm{D}$ location.

\subsection{Velocity Distribution}

Figure 9 shows the velocity contours for NPR from 1.5 to 5 for the case where no rib is attached to the wall. The values of base pressure shown in Figure 8 and the velocity contours are shown in Figure 9 are closely related. For NPR 1.5, it can be seen that the presence of oblique shock ahead of nozzle exit as the flow undergoes overexpansion causes the pattern of high and lower velocity due to expansion and compression are visible. The zone of maximum velocity occupies up to two-thirds of the duct portion. The reattachment length can be seen as blue shades where the flow recirculates, and the base pressure level is relatively low at the corner. The same zone can be seen to increase its length for NPR 2, but for higher NPR's the reattachment length is found to diminish and is very close to the base corner. The flow expands rapidly at the exit, and there is a formation of an expansion fan. The flow exiting at the nozzle, when reattached with the wall, the flow gets compressed and hence reduced velocity. The compressed fluid continues to expand at the middle of the duct, increasing velocity and compression - the strength of alternate compression and expansion increases as NPR increases. 


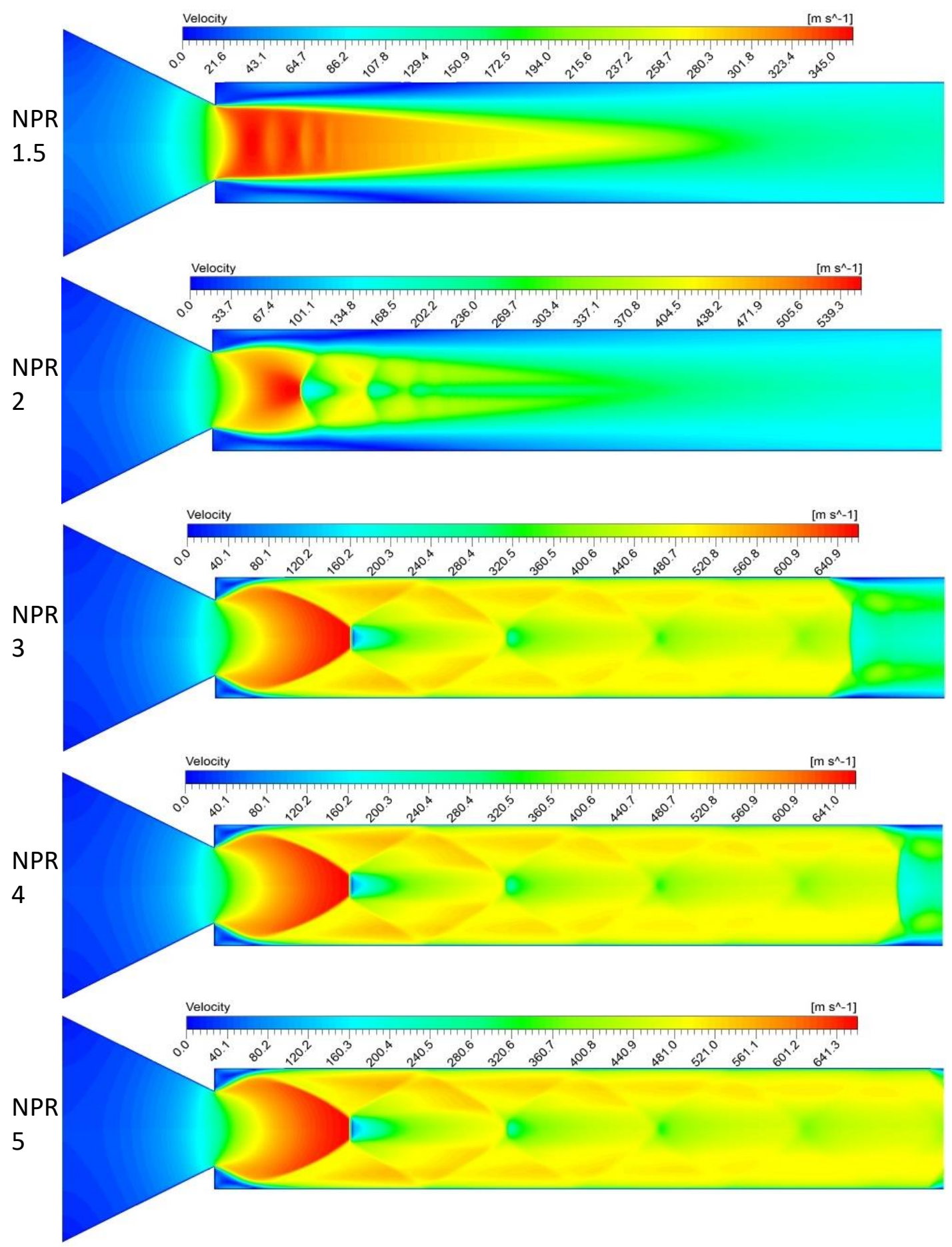

Fig. 9. Velocity contours for no rib case at various NPR

Figure 10 shows velocity distribution when a rib with an aspect ratio of $3: 1$ is attached at a location of 1D from the base corner. The NPR is increased from 1.5 to 5 . The pattern obtained is similar at NPR 1.5, and for the remaining NPR's velocity, the pattern is entirely different. Compared to velocity distribution at no rib case, the velocity is in the same range for NPR 1.5 and the base pressure obtained is nearly the same. The intermittent zones of compression and expansion did not observe for NPR 2 due to the presence of the rib. At the rib cross-section of the duct, the flow automatically gets compressed. It comes out with higher velocity, and the shear layer where the viscous dissipation is more prominent due to eddies formed by the rib causes a shift in the boundary layer. For NPR 4 and 5, these intermittent zones are lightly visible downstream of the rib. 


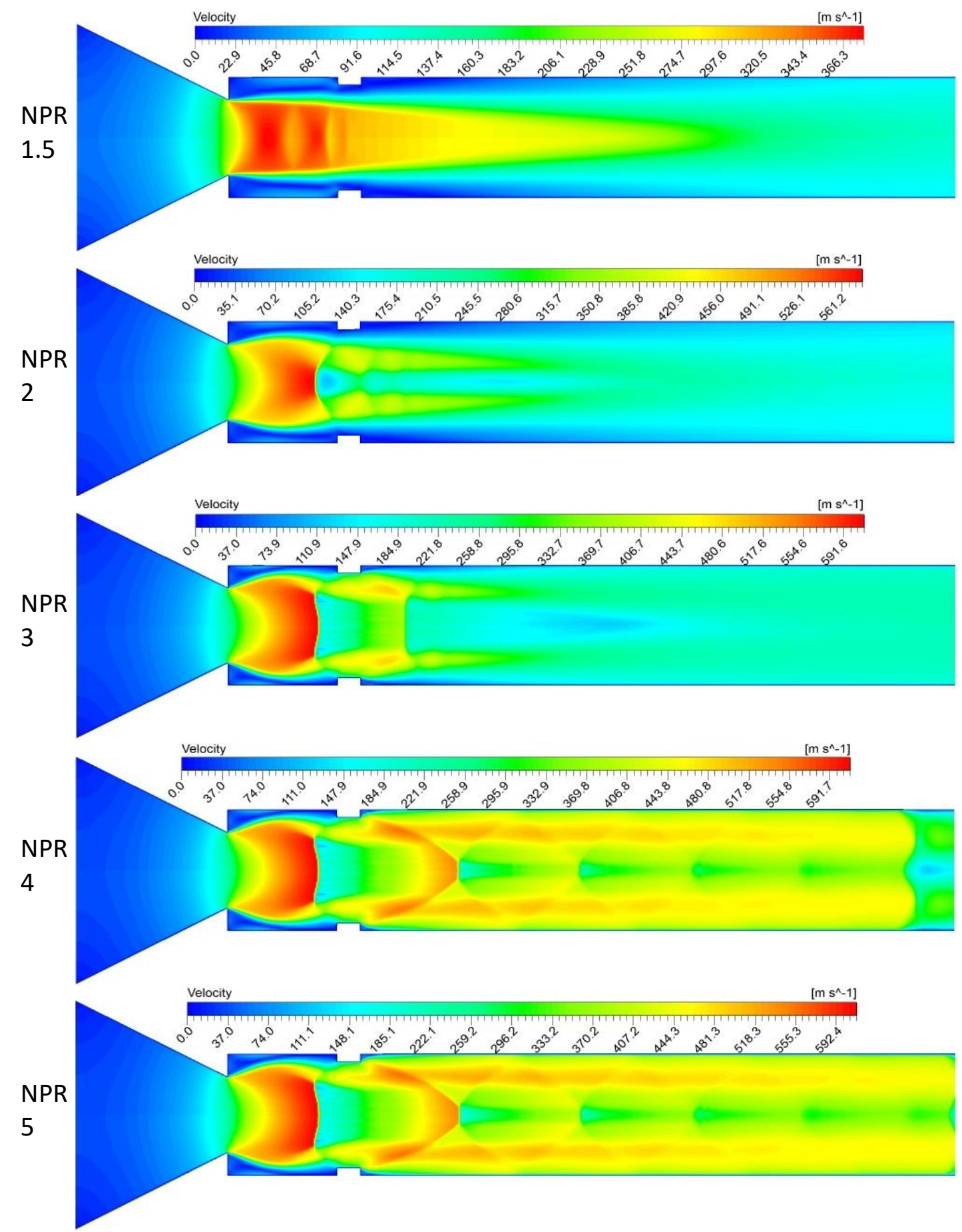

Fig. 10. Increasing outlet velocity with NPR when the rib is located at $1 D$

In Figure 11, the velocity contours are shown by shifting the single rib from 1D to 2D for NPR from 1.5 to 5 . The NPR 1.5 and 2 are the same in all the cases, so relative values of base pressure are obtained. The flow phenomena get entirely complicated for higher NPRs. When the rib is positioned at 2D for NPR 3 to 5, the viscous layer is similar. The increase in turbulent kinetic energy is more significant due to the presence of rib, as compared to duct without rib. The other compression and expansion zones are surprisingly seen only at NPR 4 and 5 in both the rib cases. Just before the rib, the flow gets reversed by a significant amount and entrained back into the mainstream through the shear layer. The shear layer is observable with a line of increased velocity. The velocity is maximum, and its distribution over the duct's entire length is found for NPR 5. For lower NPR's the velocity is 
found to be lower at the exit of the duct. For lower NPR, the velocity is found to be highest at the axial direction of the duct. As of higher NPR's, the highest velocity is at the axial centre and just ahead of the nozzle exit.



Fig. 11. Rib located at $2 \mathrm{D}$ and change in flow velocity downstream the rib

\section{Conclusions}

The present study investigates fluid flow analysis in a convergent nozzle and duct at the sonic Mach number. $K-\varepsilon$ turbulence model was used for modelling the turbulence. The flow analysis is performed numerically for different input variables. The rib influences the variation of base pressure 
in the duct. The nozzle pressure ratio (NPR) is also changed from 1.5 to 5 , while the location is fixed at $1 \mathrm{D}$ and 2D. The numerical analysis performed concludes with several significant findings. The presence of rib affects the flow regime and base pressure significantly. The rib aspect ratio of 3:2 is found to improve the base pressure significantly than with the aspect ratio of rib at $3: 1$. When the NPR is varied from 1.5 to 5 , the base pressure reduces initially for both the aspect ratios and then once the flow is under expanded, the base pressure increases at a high rate compared to the duct with no rib. When the rib location is shifted from 1D to 4D, the base pressure continuously increases. Hence maximum base pressure is obtained at an aspect ratio of $3: 2$ and the position of 4D.

\section{Acknowledgement}

Acknowledgement to Ministry of Higher Education Malaysia for Fundamental Research Grant Scheme with Project Code: FRGS/1/2019/TK07/USM/03/5.

\section{References}

[1] Ashfaq, Syed, and S. A. Khan. "The Effect of Micro Jets on Wall Pressure for Sonic under expanded Flow." International Journal of Engineering Research and Applications (IJERA) 4, no. 3 (2014): 32-38.

[2] Vikramaditya, N. S., M. Viji, S. B. Verma, Naveed Ali, and D. N. Thakur. "Base pressure fluctuations on typical missile configuration in presence of base cavity." Journal of Spacecraft and Rockets 55, no. 2 (2018): 335-345. https://doi.org/10.2514/1.A33926

[3] Schrijer, F. F. J., A. Sciacchitano, and F. Scarano. "Spatio-temporal and modal analysis of unsteady fluctuations in a high-subsonic base flow." Physics of Fluids 26, no. 8 (2014): 086101. https://doi.org/10.1063/1.4891257

[4] Jukes, Timothy, Takehiko Segawa, and Hirohide Furutani. "Active flow separation control on a NACA 4418 using DBD vortex generators and FBG sensors." In 50th AIAA aerospace sciences meeting including the new horizons forum and aerospace exposition, p. 1139. 2012. https://doi.org/10.2514/6.2012-1139

[5] Khan, Sher Afghan, and Ethirajan Rathakrishnan. "Active control of suddenly expanded flows from overexpanded nozzles." International Journal of Turbo and Jet Engines 19, no. 1-2 (2002): 119-126. https://doi.org/10.1515/TJJ.2002.19.1-2.119

[6] Khan, Ambareen, Abdul Aabid, and Sher Afghan Khan. "CFD analysis of convergentdivergent nozzle flow and base pressure control using micro-JETS." International Journal of Engineering and Technology 7, no. 3.29 (2018): 232235. https://doi.org/10.14419/ijet.v7i3.29.18802

[7] Fharukh, Ahmed GM, Abdulrehman A. Alrobaian, Abdul Aabid, and Sher Afghan Khan. "Numerical analysis of convergent-divergent nozzle using finite element method." International Journal of Mechanical and Production Engineering Research and Development 8, no. 6 (2018): 373-382. https://doi.org/10.24247/ijmperddec201842

[8] Aabid, Abdul, Ambreen Khan, Nurul Musfirah Mazlan, Mohd Azmi Ismail, Mohammad Nishat Akhtar, and Sher Afghan Khan. "Numerical simulation of suddenly expanded flow at Mach 2.2." International Journal of Engineering and Advanced Technology 8, no. 3 (2019): 457-462.

[9] Khan, Sher Afghan, Abdul Aabid, and C. Ahamed Saleel. "Influence of micro jets on the flow development in the enlarged duct at supersonic Mach number." International Journal of Mechanical and Mechatronics Engineering 19, no. 01 (2019): 70-82.

[10] Pathan, Khizar Ahmed, Prakash S. Dabeer, and Sher Afghan Khan. "Effect of nozzle pressure ratio and control jets location to control base pressure in suddenly expanded flows." Journal of Applied Fluid Mechanics 12, no. 4 (2019): 1127-1135. https://doi.org/10.29252/jafm.12.04.29495

[11] Pathan, Khizar Ahmed, Prakash S. Dabeer, and Sher Afghan Khan. "An investigation to control base pressure in suddenly expanded flows." International Review of Aerospace Engineering (I. RE. AS. E) 11, no. 4 (2018): $162-169$. https://doi.org/10.15866/irease.v11i4.14675

[12] Pathan, Khizar Ahmed, Sher Afghan Khan, and P. S. Dabeer. "CFD analysis of effect of area ratio on suddenly expanded flows." In 2017 2nd International Conference for Convergence in Technology (I2CT), pp. 1192-1198. IEEE, 2017. https://doi.org/10.1109//2CT.2017.8226315

[13] Pathan, Khizar Ahmed, Sher Afghan Khan, and P. S. Dabeer. "CFD analysis of effect of flow and geometry parameters on thrust force created by flow from nozzle." In 2017 2nd International Conference for Convergence in Technology (I2CT), pp. 1121-1125. IEEE, 2017. https://doi.org/10.1109/I2CT.2017.8226302

[14] Pathan, Khizar Ahmed, Sher Afghan Khan, and P. S. Dabeer. "CFD analysis of effect of Mach number, area ratio and nozzle pressure ratio on velocity for suddenly expanded flows." In 2017 2nd International Conference for Convergence in Technology (I2CT), pp. 1104-1110. IEEE, 2017. https://doi.org/10.1109/I2CT.2017.8226299 
[15] Pathan, Khizar Ahmed, Prakash S. Dabeer, and Sher Afghan Khan. "Investigation of base pressure variations in internal and external suddenly expanded flows using CFD analysis." CFD Letters 11, no. 4 (2019): 32-40.

[16] Khan, Ambareen, Nurul Musfirah Mazlan, Mohd Azmi Ismail, and Mohammad Nishat Akhtar. "Experimental and numerical simulations at sonic and supersonic Mach numbers for area ratio 7.84." CFD Letters 11, no. 5 (2019): 50 60.

[17] Khan, Ambareen, Nurul Musfirah Mazlan, and Mohd Azmi Ismail. "Analysis of flow through a convergent nozzle at Sonic Mach Number for Area Ratio 4." Journal of Advanced Research in Fluid Mechanics and Thermal Sciences 62, no. 1 (2019): 66-79.

[18] Khan, Ambareen, Nurul Musfirah Mazlan, and Mohd Azmi Ismail. "Investigation of the flowfield at sonic and supersonic mach numbers with sudden expansion." Int. J. Innov. Technol. Explor. Eng 8, no. 6S3 (2019): 91-95.

[19] Khan, Ambareen, Mohd Azmi Ismail, and Nurul Musfirah Mazlan. "Numerical Simulation of Suddenly Expanded Flow from Converging Nozzle at Sonic Mach Number." In Proceedings of International Conference of Aerospace and Mechanical Engineering 2019, pp. 349-359. Springer, Singapore, 2020. https://doi.org/10.1007/978-981-15-4756$\underline{029}$

[20] Khan, Ambareen, Parvathy Rajendran, and Junior Sarjit Singh Sidhu. "Passive Control of Base Pressure: A Review." Applied Sciences 11, no. 3 (2021): 1334. https://doi.org/10.3390/app11031334

[21] Rathakrishnan, E. "Effect of ribs on suddenly expanded flows." AIAA journal 39, no. 7 (2001): 1402-1404. https://doi.org/10.2514/2.1461 